\title{
Arzneipflanzen in der Stillzeit - eine Bewertung
}

\author{
Beatrix Falch \\ Fachgruppe Phytopharmazie, Zürcher Hochschule für Angewandte Wissenschaften, Wädenswil, Schweiz
}

Die Verabreichung von Arzneimitteln an stillende Mütter hat immer unter der Voraussetzung zu geschehen, dass der Säugling nicht gefährdet werden darf. Auch wenn das Risiko, die Gesundheit des Säuglings durch eine Medikation zu beeinträchtigen, deutlich geringer ist als während der Schwangerschaft, muss dem Umstand Rechnung getragen werden, dass eine Vielzahl von Arzneimitteln via Muttermilch zum Säugling gelangen kann. Stillende Mütter ziehen daher mehrheitlich pflanzliche oder sogenannte «natürliche Arzneimittel» den schulmedizinischen Medikamenten vor. Mit wenigen Ausnahmen fehlen aber Studien zur Anwendung von Arzneipflanzen und deren Zubereitungen in der Stillzeit. So stellt sich die Frage, wie die einzelnen pflanzlichen Zubereitungen bezüglich ihrer Anwendbarkeit in der Stillzeit zu bewerten sind.

Die Autorin hat nun anhand des vorhandenen wissenschaftlichen Datenmaterials und Erfahrungswissens eine Bewertung von Arzneipflanzen zur Anwendung in Schwangerschaft und Stillzeit vorgenommen, um Ärzten und Apothekern eine Entscheidungshilfe an die Hand zu geben, die es ermöglicht, Empfehlungen auszusprechen.

\section{Arzneipflanzenbewertung}

Dazu wurden klinische, pharmakologische, toxikologische und analytische Daten - sofern vorhanden - analysiert und interpretiert, um das Gefährdungspotenzial jeder einzelnen pflanzlichen Zubereitung einschätzen zu können. Hierfür wurde gezielt nach Publikationen in Datenbanken wie PubMed gesucht, und die Assessment Reports des Committee on Herbal Medicinal Products (HMPC) der europäischen Arzneimittelbehörde (EMA) wurden konsultiert [1].

\section{Datenbank «Herba pro Matre»}

Die daraus gewonnene Bewertung wurde dann mit dem dokumentierten traditionellen Wissen sowie den Praxiserfahrungen aus verschiedenen Diskussionsforen sowie der Literatur [2-8] verglichen. Daraus ergab sich eine differen- zierte Beurteilung, die meist von den offiziell zur Verfügung stehenden Monographien [3, 9, 10] abweicht, da aufgrund des Fehlens klinischer Studien den Praxiserfahrungen eine grosse Bedeutung beigemessen wurde. Diese Bewertungen finden sich in der Datenbank «Herba pro Matre» (www. phytocura.ch/Phytocura/Datenbank.html).

Im Nachfolgenden werden beispielhaft phytotherapeutische Möglichkeiten zur Behandlung von Beschwerden im Wochenbett und in der Stillzeit vorgestellt. Zusammengefasst findet sich die entsprechende Arzneipflanzenliste in Tabelle 1.

\section{Arzneipflanzen in der Stillzeit}

Im Wochenbett ist der Eisen- und Calciumbedarf erhöht. Neben entsprechenden diätetischen Massnahmen kann zusätzlich mit Brennnesselkraut und Löwenzahnblättern als Tee oder Tinktur die Eisen- und Calciumaufnahme aus der Nahrung gefördert werden.

Die Rückbildung der Gebärmutter wird traditionell mit Gänseblümchenkraut als Urtinktur unterstützt. Weitere bewährte Arzneipflanzen sind Frauenmantelkraut, Himbeerblätter und Schafgarbe, die zusätzlich auch noch eine blutstillende Wirkung haben. Ebenfalls eine blutstillende Wirkung hat das Hirtentäschelkraut. Diese Arzneipflanzen können alle als Tees oder (Ur-)Tinkturen, am besten in Kombination, eingenommen werden.

Zur Linderung der Schmerzen und zur Förderung der Wundheilung nach einem Dammschnitt oder Dammriss haben sich kalte Calendula-Kompressen oder Sitzbäder mit Hamameliswasser, Hamamelisrinde oder Kamillenblütentee bewährt.

Stress oder seelische Belastungen im Wochenbett können mit beruhigend wirkenden Arzneipflanzen wie Melissenblättern, Hopfenzapfen oder Herzgespannkraut als Tee oder Tinktur, einzeln oder in Kombination, therapiert werden. Wochenbettdepressionen lassen sich erfolgreich mit Johanniskrautpräparaten therapieren (vorzugsweise nach dem Stillen einnehmen).

\section{KARGER}

Fax +497614520714

Information@Karger.com

www.karger.com
Dr. sc. nat. Beatrix Falch

Fachgruppe Phytopharmazie

Zürcher Hochschule für angewandte Wissenschaften ZHAW

Postfach, 8820 Wädenswil, Schweiz

beatrix.falch@zhaw.ch

www.zhaw.ch / www.smgp.ch 
Tab. 1. Arzneipflanzenliste für Wochenbett und Stillzeit

\begin{tabular}{|c|c|c|}
\hline Droge & Botanischer Name & Indikation \\
\hline Anisfrüchte & Pimpinella anisum & Anregung der Milchbildung \\
\hline Beinwellblätter, -wurzeln & Symphytum officinalis & Brustwarzenentzündung \\
\hline Brennnesselblätter & Urtica dioica, $U$. urens & Anregung der Milchbildung, Eisenmangel \\
\hline Dillfrüchte & Anethum graveolens & Anregung der Milchbildung \\
\hline Eisenkraut & Verbena officinalis & Anregung der Milchbildung \\
\hline Fenchelfrüchte & Foeniculum vulgare & Anregung der Milchbildung \\
\hline Frauenmantelkraut & Alchemilla vulgaris & $\begin{array}{l}\text { Blutstillung im Wochenbett, } \\
\text { Brustwarzenentzündung }\end{array}$ \\
\hline Gänseblümchenkraut & Bellis perennis & Gebärmutterrückbildung \\
\hline Geissrautenkraut & Galega officinalis & Anregung der Milchbildung \\
\hline Herzgespannkraut & Leonurus cardiaca & Beruhigung \\
\hline Himbeerblätter & Rubus idaeus & Blutstillung im Wochenbett \\
\hline Hirtentäschelkraut & Capsella bursa-pastoris & Blutstillung im Wochenbett \\
\hline Hopfenzapfen & Humulus lupulus & Beruhigung, Schlafförderung \\
\hline Johanniskraut & Hypericum perforatum & Wochenbettdepression \\
\hline Kamillenblüten & Matricaria chamomilla & Wundheilung nach Dammschnitt \\
\hline Kümmelfrüchte & Carum carvi & Anregung der Milchbildung \\
\hline Majoranblätter & Origanum vulgare & Anregung der Milchbildung \\
\hline Melissenblätter & Melissa officinalis & $\begin{array}{l}\text { Anregung der Milchbildung, Beruhigung, } \\
\text { Schlafförderung }\end{array}$ \\
\hline Pfefferminzblätter & Mentha $x$ piperita & Reduktion der Milchmenge \\
\hline Ringelblumenblüten & Calendula officinalis & $\begin{array}{l}\text { Wundheilung nach Dammschnitt, } \\
\text { Brustwarzenentzündung }\end{array}$ \\
\hline Salbeiblätter & Salvia officinalis/S. triloba & $\begin{array}{l}\text { Reduktion der Milchmenge, } \\
\text { Brustwarzenentzündung }\end{array}$ \\
\hline Schafgarbenblätter & Achillea millefolium & $\begin{array}{l}\text { Blutstillung im Wochenbett, } \\
\text { Brustwarzenentzündung }\end{array}$ \\
\hline $\begin{array}{l}\text { Zaubernussrinde/ } \\
\text { Hamameliswasser }\end{array}$ & Hamamelis virginiana & $\begin{array}{l}\text { Wundheilung nach Dammschnitt, } \\
\text { Brustwarzenentzündung }\end{array}$ \\
\hline
\end{tabular}

Zur Anregung der Milchbildung gibt es diverse TeeRezepturen, die in unterschiedlichen Kombinationen Anisfrüchte, Dillfrüchte, Fenchelfrüchte, Kümmelfrüchte, Brennnesselblätter, Eisenkraut, Geissrautenkraut, Wilder Majoran oder Melisse enthalten. Pfefferminz- oder Salbeiblätter eignen sich dagegen zur Reduktion der Milchmenge.

Bei entzündeten Brustwarzen empfiehlt sich eine Kompresse oder Salbe aus Ringelblumenblüten, Beinwellblättern, Beinwellwurzeln, Schafgarbenkraut oder Frauenmantelkraut nach dem Stillen oder das direkte Betupfen der Brustwarzen mit Hamameliswasser, Salbeitee oder Schwarztee nach dem Stillen (danach unbedingt fetten).

Da nicht alle genannten Arzneidrogen auch als Fertigpräparate vorliegen, empfiehlt es sich, diese als Magistralrezepturen zu verordnen.

\section{Dank}

Die Autorin dankt Dr. med. Cesare Canonica für die grosse technische Unterstützung bei der Erstellung der Datenbank «Herba pro Matre» sowie allen aktiven Teilnehmerinnen des Phyto-Gyni-Netzwerks HERBADONNA für ihre wertvollen phytotherapeutischen Erfahrungen.

\section{Online Supplemental Material}

Die Literatur findet sich im Tagungsband und kann auf www.smgp.ch/ smgp/homeindex/jahrestagung.html heruntergeladen werden.

\section{Disclosure Statement}

Es besteht kein Interessenkonflikt. 